\title{
Combination of Partial Stochastic Linearization and Karhunen-Loeve Expansion to Design Coriolis Dynamic Vibration Absorber
}

\author{
Viet Duc La \\ Institute of Mechanics, Vietnam Academy of Science and Technology, 264 Doi Can, Hanoi, Vietnam \\ Correspondence should be addressed to Viet Duc La; ldviet@imech.vast.vn
}

Received 22 January 2017; Accepted 23 March 2017; Published 20 April 2017

Academic Editor: Denis Benasciutti

Copyright (C) 2017 Viet Duc La. This is an open access article distributed under the Creative Commons Attribution License, which permits unrestricted use, distribution, and reproduction in any medium, provided the original work is properly cited.

Coriolis dynamic vibration absorber is a device working in nonlinear zone. In stochastic design of this device, the Monte Carlo simulation requires large computation time. A simplified model of the system is built to retain the most important nonlinear term, the Coriolis damping of the dynamic vibration absorber. Applying the full equivalent linearization technique to the simplified model is inaccurate to describe the nonlinear behavior. This paper proposes a combination of partial stochastic linearization and Karhunen-Loeve expansion to solve the problem. The numerical demonstration of a ropeway gondola induced by wind load is presented. A design example based on the partial linearization supports the advantage of the proposed approach.

\section{Introduction}

Dynamic vibration absorber (DVA) or tuned mass damper composed of mass, spring, and damper is widely used to suppress vibration. The theory of linear DVA is well developed in literature [1] for a variety of excitations of interest, including the random excitation. In pendulum structures such as ropeway gondola or floating structures (e.g., ships or tension leg platforms), using DVA reveals several surprising and interesting facts not met in normal systems, including location problem, Coriolis force, and gyroscopic moment [2-8]. The Coriolis DVA has been studied in the cases of harmonic and free vibrations $[5,6]$ but not the case of random vibration. In fact, the environmental loads acting on pendulum structures, such as wind or wave, are random in nature and the deterministic approaches in some cases are not enough to assess the adequacy of a design. The Coriolis DVA [5] only works with nonlinear vibration. The pendulum structure attached with Coriolis DVA is nonlinear and has four states. It is well known that only Monte Carlo simulation and equivalent linearization are suited to solve the stochastic dynamics problem of nonlinear system with the state-space dimension larger than 4 [9]. The Monte Carlo simulation has particular advantages but it requires time-consuming work and thereby it is not very suited for stochastic design. On the other hand, the equivalent linearization based on the assumption of Gaussian distributed responses is much simpler and well developed in literature [9-12]. However, in this paper, we will show that the full equivalent linearization technique hides some important nonlinearity and thereby this method is totally inadequate to analyze the system.

In our recent study [13], the so-called partial linearization has been developed for a spherical pendulum with the radial spring-damper. However, the partial linearization alone as presented in [13] can only solve the case of white noise excitation. The wind or wave excitation in fact is modeled by some spectrums which are far from white noise. Therefore, in this paper, we propose a combination of the partial linearization and the Karhunen-Loeve (KL) expansion to solve this problem. The KL expansion is a well-known method to represent stochastic process [14]. For instance, it has been used in finite element method [14], in buckling analysis [15, 16], or in reliability analysis [17]. For the specific system in this paper, the orthonormality relation of KL expansion is quite useful to realize the partial linearization technique.

The novelty of this paper is the proposal of partial linearization technique combined with KL expansion. This approach is much more accurate than the full linearization 


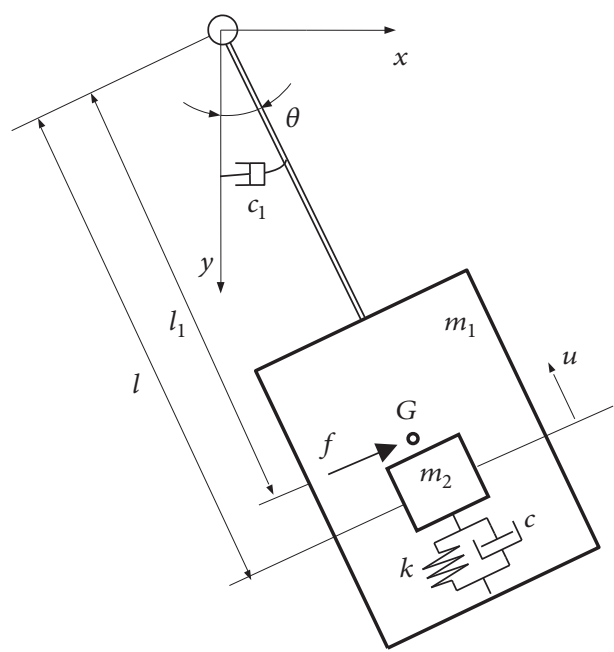

Figure 1: Pendulum structure with a Coriolis dynamic vibration absorber.

technique while the time consumed is much shorter than the Monte Carlo simulation. The structure of paper is as follows. Firstly, in Section 2, the nonlinear motion equation is written in nondimensional form and is simplified with some adequate assumptions. In Section 3, the full linearization and partial linearization techniques are presented to show that the full linearization is inadequate to describe the effect of the second-order terms. In Section 4, the KL expansion is used to realize the partial linearization method. Lastly, in Section 5, the accuracy of the proposed approach is verified by Monte Carlo simulation and a design example is presented.

\section{Motion Equations}

2.1. Original Motion Equations. In Figure 1, let us consider a pendulum structure having a concentrated mass $m_{1}$ and a pendulum length $l_{1}$.

$c_{1}$ is the structural damping coefficient, $\theta$ is the rotational angle of the pendulum, $u$ is the displacement of the DVA measured from the static position, $l$ is the distance between the fulcrum and the DVA in the static condition, $g$ is the acceleration of gravity, and $m_{2}, k$, and $c$ are the mass, spring constant, and damping coefficient of the DVA, respectively.

Assume that the pendulum structure is subjected to a random external force $f(t)$. It is not difficult to derive the motion equations as follows [5]:

$$
\begin{aligned}
& m_{1} l_{1}^{2} \ddot{\theta}+m_{2}(l-u)^{2} \ddot{\theta}+c_{1} \dot{\theta}-2 m_{2}(l-u) \dot{u} \dot{\theta} \\
& \quad+\left(m_{1} g l_{1}+m_{2} g l-m_{2} g u\right) \sin \theta=l_{1} f(t), \\
& m_{2} \ddot{u}+c \dot{u}+k u+m_{2}(l-u) \dot{\theta}^{2}-m_{2} g(1-\cos \theta)=0 .
\end{aligned}
$$

The following parameters are introduced:

$$
\begin{aligned}
& \omega_{s}=\sqrt{\frac{g}{l_{1}}} ; \\
& \zeta_{s}=\frac{c_{1}}{2 l_{1}^{2} m_{1} \omega_{s}} ;
\end{aligned}
$$

$$
\begin{aligned}
\tau & =\omega_{s} t ; \\
\mu & =\frac{m_{2}}{m_{1}} ; \\
\alpha & =\frac{\sqrt{k / m_{2}}}{\omega_{s}} ; \\
\zeta & =\frac{c}{2 m_{2} \omega_{s}} ; \\
\gamma & =\frac{l}{l_{1}} ; \\
z & =\frac{u}{l_{1}}
\end{aligned}
$$

in which $\omega_{s}$ and $\zeta_{s}$, respectively, are the natural frequency and the damping ratio of the main structure, $\tau$ is the nondimensional time with time scale $\omega_{s}^{-1}, \mu$ is the DVA mass ratio, $\alpha$ is the DVA frequency ratio, $\zeta$ is the DVA damping ratio, $\gamma$ specifies the position of the DVA, and $z$ is the nondimensional form of DVA displacement. The motion equations (1) are written in the nondimensional forms as follows:

$$
\begin{aligned}
& \left\{1+\mu(\gamma-z)^{2}\right\} \ddot{\theta}+2 \zeta_{s} \dot{\theta}-2 \mu(\gamma-z) \dot{z} \dot{\theta} \\
& \quad+\{1+\mu(\gamma-z)\} \sin \theta=\frac{f(\tau)}{\left(m_{1} g\right)}, \\
& \ddot{z}+2 \zeta \dot{z}+\alpha^{2} z+(\gamma-z) \dot{\theta}^{2}-(1-\cos \theta)=0,
\end{aligned}
$$

in which the dot operator from now denotes the differentiation with respect to normalized time $\tau$. Equations (3) and (4) are used in Monte Carlo simulations in Section 5. Before moving further, we can draw an important property of the motion equations as follows. In (4), the centrifugal force $(\gamma-$ $z) \dot{\theta}^{2}$ and the gravity force $(1-\cos \theta)$ are the driving forces on the DVA. These terms have frequency of about two times the frequency of $\theta$. Therefore, the resonant frequency of the DVA is about twice that of the pendulum.

2.2. Simplified Equations. The stochastic differential equations (3) and (4) are quite complicated to apply any equivalent linearization technique. We should do some simplifications. As seen from (3) and (4), three sources of system nonlinearity are due to the variation of the arm length $\gamma-z$, the Coriolis damping $\dot{z} \dot{\theta}$, and the trigonometric functions. Considering full sources of nonlinearity in a general system is an important development in the future studies. In this paper, to illustrate the effectiveness of the proposed combination, we make the following assumptions.

(i) The effect of the variation of the arm length is ignored, such as

$$
\gamma-z \approx \gamma
$$

The approximations are appropriate because, in the practical application, the DVA normalized displacement $z$ is small 
enough in comparison with the DVA location parameter $\gamma$. However, as stated above, in the future studies, the variation of the arm length should be taken into account.

(ii) The nonlinearity of the trigonometric functions is retained up to the second order, such as

$$
\begin{aligned}
& \sin \theta \approx \theta, \\
& \cos \theta \approx 1-\frac{\theta^{2}}{2} .
\end{aligned}
$$

These approximations produce acceptable errors $(<5 \%)$ for the angle up to 30 degrees.

Equations (3) and (4) become

$$
\begin{gathered}
\left(1+\mu \gamma^{2}\right) \ddot{\theta}+2 \zeta_{s} \dot{\theta}-2 \mu \gamma \dot{z} \dot{\theta}+(1+\mu \gamma) \theta=\frac{f(\tau)}{\left(m_{1} g\right)}, \\
\ddot{z}+2 \zeta \dot{z}+\alpha^{2} z+\gamma \dot{\theta}^{2}-\frac{\theta^{2}}{2}=0 .
\end{gathered}
$$

In the next sections, the linearization techniques are discussed in the simplified equation (7).

\section{Equivalent Linearization Techniques}

3.1. Full Linearization. The simplest equivalent linearization technique is based on the assumption of Gaussian distributed responses $[10,11]$. Consider the state vector defined as

$$
\mathbf{x}(\tau)=\left[\begin{array}{llll}
\theta & \dot{\theta} & z & \dot{z}
\end{array}\right]^{T}
$$

Any nonlinear vector function $\mathbf{g}(\mathbf{x})$ can be linearized by $[9,11,12]$

$$
\mathbf{g}(\mathbf{x}) \approx\langle\mathbf{g}(\mathbf{x})\rangle+\mathbf{B}(\tau)(\mathbf{x}(\tau)-\langle\mathbf{x}(\tau)\rangle)
$$

in which $\langle\bullet\rangle$ denotes the averaging operator while the components of the matrix $\mathbf{B}(\tau)$ of the linearization coefficients are determined by

$$
B_{j l}(\tau)=\left\langle\frac{\partial}{\partial x_{l}} g_{j}(\mathbf{x})\right\rangle .
$$

Applying (9) and (10) to the nonlinear functions in (7), we have

$$
\begin{aligned}
\dot{z} \dot{\theta} & \approx\langle\dot{z} \dot{\theta}\rangle+\langle\dot{z}\rangle(\dot{\theta}-\langle\dot{\theta}\rangle)+\langle\dot{\theta}\rangle(\dot{z}-\langle\dot{z}\rangle), \\
\dot{\theta}^{2} & \approx\left\langle\dot{\theta}^{2}\right\rangle+2\langle\dot{\theta}\rangle(\dot{\theta}-\langle\dot{\theta}\rangle), \\
\theta^{2} & \approx\left\langle\theta^{2}\right\rangle+2\langle\theta\rangle(\theta-\langle\theta\rangle) .
\end{aligned}
$$

Substituting (11) into (7) and rearranging give the linearized equation in matrix form:

$$
\begin{aligned}
{\left[\begin{array}{cc}
1+\mu \gamma^{2} & 0 \\
0 & 1
\end{array}\right]\left[\begin{array}{l}
\ddot{\theta} \\
\ddot{z}
\end{array}\right] } \\
+\left[\begin{array}{cc}
2\left(\zeta_{s}-\mu \gamma\langle\dot{z}\rangle\right) & -2 \mu \gamma\langle\dot{\theta}\rangle \\
2 \gamma\langle\dot{\theta}\rangle & 2 \zeta
\end{array}\right]\left[\begin{array}{l}
\dot{\theta} \\
\dot{z}
\end{array}\right] \\
+\left[\begin{array}{cc}
1+\mu \gamma & 0 \\
-\langle\theta\rangle & \alpha^{2}
\end{array}\right]\left[\begin{array}{l}
\theta \\
z
\end{array}\right] \\
=\left[\begin{array}{c}
2 \mu \gamma(\langle\dot{z} \dot{\theta}\rangle-2\langle\dot{z}\rangle\langle\dot{\theta}\rangle) \\
\gamma\left(2\langle\dot{\theta}\rangle^{2}-\left\langle\dot{\theta}^{2}\right\rangle\right)+\frac{\left\langle\theta^{2}\right\rangle}{2}-\langle\theta\rangle^{2}
\end{array}\right] \\
+\left[\begin{array}{c}
\frac{f(\tau)}{\left(m_{1} g\right)} \\
0
\end{array}\right] .
\end{aligned}
$$

An important remark is drawn from (12). In the stationary case, the average values $\langle\dot{z}\rangle,\langle\dot{\theta}\rangle$ must be equal to zeros. In this case, the first row of (12) will show that the Coriolis DVA has no dynamic effect at all. This phenomenon does not agree with the Monte Carlo simulation results in Section 5. That means the full linearization technique applied to the simplified equation (7) hides some important nonlinearity describing the DVA's effect.

3.2. Partial Linearization. The full linearization technique linearizes the system too much that hides some important nonlinearities. The partial linearization is considered instead. The idea has been studied in [13] and is presented here for convenience. The effective damping approach is used to approximate the Coriolis term $\dot{z} \dot{\theta}$ in the first equation of (7). The Coriolis term is replaced by the linear damping as

$$
\dot{z} \dot{\theta} \approx-c_{e} \dot{\theta}
$$

in which $c_{e}$ is the effective damping coefficient. The minus sign is used to make $c_{e}$ be positive. The damping coefficient is chosen to minimize the following error:

$$
E=\left\langle\left(\dot{z} \dot{\theta}+c_{e} \dot{\theta}\right)^{2}\right\rangle
$$
gives

Setting the derivative of $E$ with respect to $c_{e}$ equal to zero

$$
c_{e}=\frac{-\left\langle\dot{z} \dot{\theta}^{2}\right\rangle}{\left\langle\dot{\theta}^{2}\right\rangle} \text {. }
$$


Substituting the replacements (13) and (15) into (7) gives

$$
\begin{aligned}
& \left(1+\mu \gamma^{2}\right) \ddot{\theta}+2\left(\zeta_{s}-\mu \gamma \frac{\left\langle\dot{z} \dot{\theta}^{2}\right\rangle}{\left\langle\dot{\theta}^{2}\right\rangle}\right) \dot{\theta}+(1+\mu \gamma) \theta \\
& =\frac{f(\tau)}{m_{1} g}, \\
& \ddot{z}+2 \zeta \dot{z}+\alpha^{2} z+\gamma \dot{\theta}^{2}-\frac{\theta^{2}}{2}=0 .
\end{aligned}
$$

The first equation of (16) is linearized while the second is still nonlinear. Therefore, we call this technique partial linearization. This partial linearization can keep the important property of the resonant frequency of the DVA. The problem, which the partial linearization has to face, is the difficulty in calculating the stochastic nonlinear responses. In the next section, we show that the orthonormality relation of the KL expansion is quite useful to realize the proposed partial linearization technique.

\section{Implementation of Karhunen-Loeve Expansion}

The KL expansion is well known in literature. The orthonormality relation of the $\mathrm{KL}$ expansion is presented in the Appendix. Let us discuss the details of the implementation of KL expansion to the linearization techniques presented in Section 3. Assume that the external excitation $f(\tau)$ is Gaussian; it can be expressed by KL expansion as

$$
f(\tau)=f_{0}+\sum_{j=1}^{N} \xi_{j} \sqrt{\lambda_{j}} f_{j}(\tau)
$$

in which $N$ is the order of the truncation of KL expansion, $f_{0}=\langle f\rangle, f_{j}$ are the eigenfunctions of the covariance kernel, where $\lambda_{j}$ are the associated eigenvalues, and $\xi_{j}$ are the uncorrelated Gaussian random variables with zero mean values and unit variance, such as

$$
\begin{aligned}
\left\langle\xi_{j}\right\rangle & =0, \\
\left\langle\xi_{i} \xi_{j}\right\rangle & =\delta_{i j} \\
& (i, j=1, \ldots, N)
\end{aligned}
$$

in which $\delta$ denotes the Dirac delta function. Because the partial linearization technique relates to the higher order statistics of the response, we need more orthonormality relations of random variables $\xi_{j}$. In the Appendix, we show the following relations:

$$
\begin{aligned}
\left\langle\xi_{i} \xi_{j} \xi_{k}\right\rangle & =0, \\
\left\langle\xi_{i} \xi_{j} \xi_{k} \xi_{l}\right\rangle & =\delta_{i j} \delta_{k l}+\delta_{i k} \delta_{j l}+\delta_{i l} \delta_{j k}
\end{aligned}
$$

The relations (19) are useful to realize partial linearization technique.

4.1. Implementation of KL Expansion to Full Linearization. The solutions of full linearized equation (12) can be expressed by

$$
\begin{aligned}
& \theta=\theta_{0}+\sum_{j=1}^{N} \xi_{j} \theta_{j} ; \\
& \dot{\theta}=\dot{\theta}_{0}+\sum_{j=1}^{N} \xi_{j} \dot{\theta}_{j} ; \\
& \ddot{\theta}=\ddot{\theta}_{0}+\sum_{j=1}^{N} \xi_{j} \ddot{\theta}_{j}, \\
& z=z_{0}+\sum_{j=1}^{N} \xi_{j} z_{j}, \\
& \dot{z}=\dot{z}_{0}+\sum_{j=1}^{N} \xi_{j} \dot{z}_{j} ; \\
& \ddot{z}=\ddot{z}_{0}+\sum_{j=1}^{N} \xi_{j} \ddot{z}_{j},
\end{aligned}
$$

in which the KL functions of the response are calculated from

$$
\begin{aligned}
& {\left[\begin{array}{cc}
1+\mu \gamma^{2} & 0 \\
0 & 1
\end{array}\right]\left[\begin{array}{l}
\ddot{\theta}_{j} \\
\ddot{z}_{j}
\end{array}\right]} \\
& +\left[\begin{array}{cc}
2\left(\zeta_{s}-\mu \gamma\langle\dot{z}\rangle\right) & -2 \mu \gamma\langle\dot{\theta}\rangle \\
2 \gamma\langle\dot{\theta}\rangle & 2 \zeta
\end{array}\right]\left[\begin{array}{c}
\dot{\theta}_{j} \\
\dot{z}_{j}
\end{array}\right] \\
& +\left[\begin{array}{cc}
1+\mu \gamma & 0 \\
-\langle\theta\rangle & \alpha^{2}
\end{array}\right]\left[\begin{array}{c}
\theta_{j} \\
z_{j}
\end{array}\right] \\
& =\left[\begin{array}{c}
2 \mu \gamma(\langle\dot{z} \dot{\theta}\rangle-2\langle\dot{z}\rangle\langle\dot{\theta}\rangle) \\
\gamma\left(2\langle\dot{\theta}\rangle^{2}-\left\langle\dot{\theta}^{2}\right\rangle\right)+\frac{\left\langle\theta^{2}\right\rangle}{2}-\langle\theta\rangle^{2}
\end{array}\right]+\left[\begin{array}{c}
\left(f_{j}(\tau)\right. \\
\left(m_{1} g\right)
\end{array}\right]
\end{aligned}
$$

The mean values are calculated as

$$
\begin{aligned}
\langle\dot{z}\rangle & =\sum_{j=0}^{N} \dot{z}_{j} ; \\
\langle\dot{\theta}\rangle & =\sum_{j=0}^{N} \dot{\theta}_{j} ;
\end{aligned}
$$




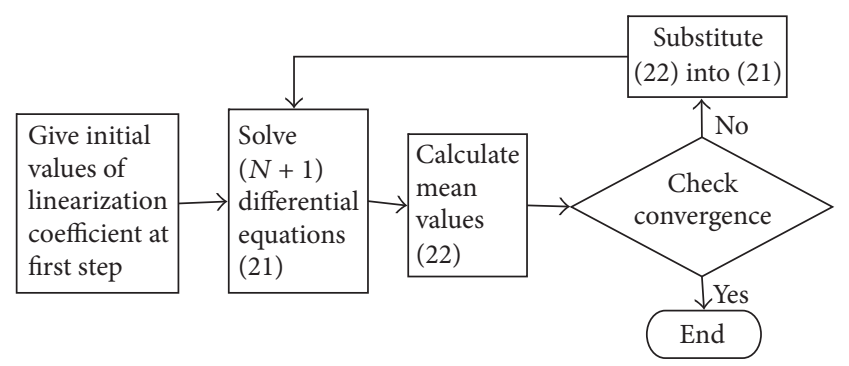

Figure 2: Flowchart of implementation of KL expansion to full linearization technique.

$$
\begin{aligned}
\langle\theta\rangle & =\sum_{j=0}^{N} \theta_{j} ; \\
\langle\dot{z} \dot{\theta}\rangle & =\sum_{j=0}^{N} \dot{z}_{j} \dot{\theta}_{j} ; \\
\left\langle\dot{\theta}^{2}\right\rangle & =\sum_{j=0}^{N} \dot{\theta}_{j}^{2} ; \\
\left\langle\theta^{2}\right\rangle & =\sum_{j=0}^{N} \theta_{j}^{2} .
\end{aligned}
$$

The iterative procedure of the implementation of $\mathrm{KL}$ expansion to full linearization technique is shown in Figure 2.

\subsection{Implementation of KL Expansion to Partial Linearization.} Because (16) is only partially linearized, the implementation of KL expansion is more complicated. The solution of the first equation of (16) can be expressed by

$$
\begin{aligned}
& \theta=\theta_{0}+\sum_{j=1}^{N} \xi_{j} \theta_{j} ; \\
& \dot{\theta}=\dot{\theta}_{0}+\sum_{j=1}^{N} \xi_{j} \dot{\theta}_{j} ; \\
& \ddot{\theta}=\ddot{\theta}_{0}+\sum_{j=1}^{N} \xi_{j} \ddot{\theta}_{j}
\end{aligned}
$$

in which the KL functions of the response are calculated from

$$
\begin{aligned}
& \left(1+\mu \gamma^{2}\right) \ddot{\theta}_{j}+2\left(\zeta_{s}-\mu \gamma \frac{\left\langle\dot{z}^{2}\right\rangle}{\left\langle\dot{\theta}^{2}\right\rangle}\right) \dot{\theta}_{j}+(1+\mu \gamma) \theta_{j} \\
& =\frac{f_{j}(\tau)}{m_{1} g} \quad(j=0,1, \ldots, N) .
\end{aligned}
$$

Substituting (23) into the second equation of (16) gives

$$
\begin{gathered}
\ddot{z}+2 \zeta \dot{z}+\alpha^{2} z+\gamma\left(\dot{\theta}_{0}+\sum_{j=1}^{N} \xi_{j} \dot{\theta}_{j}\right)^{2} \\
-\frac{\left(\theta_{0}+\sum_{j=1}^{N} \xi_{j} \theta_{j}\right)^{2}}{2}=0 .
\end{gathered}
$$

Equation (25) can be rearranged as

$$
\begin{gathered}
\ddot{z}+2 \zeta \dot{z}+\alpha^{2} z+\gamma \dot{\theta}_{0}^{2}-\frac{\theta_{0}^{2}}{2}+\sum_{j=1}^{N} \xi_{j}\left(2 \gamma \dot{\theta}_{0} \dot{\theta}_{j}-\theta_{0} \theta_{j}\right) \\
+\sum_{i=1}^{N} \sum_{j=1}^{N} \xi_{i} \xi_{j}\left(\gamma \dot{\theta}_{i} \dot{\theta}_{j}-\frac{\theta_{i} \theta_{j}}{2}\right)=0
\end{gathered}
$$

This rearrangement yields the following form of DVA response:

$$
\begin{aligned}
& z=z_{00}+\sum_{j=1}^{N} \xi_{j} z_{0 j}+\sum_{i=1}^{N} \sum_{j=1}^{N} \xi_{i} \xi_{j} z_{i j} ; \\
& \dot{z}=\dot{z}_{00}+\sum_{j=1}^{N} \xi_{j} \dot{z}_{0 j}+\sum_{i=1}^{N} \sum_{j=1}^{N} \xi_{i} \xi_{j} \dot{z}_{i j} ; \\
& \ddot{z}=\ddot{z}_{00}+\sum_{j=1}^{N} \xi_{j} \ddot{z}_{0 j}+\sum_{i=1}^{N} \sum_{j=1}^{N} \xi_{i} \xi_{j} \ddot{z}_{i j}
\end{aligned}
$$

in which the KL functions are calculated from

$$
\begin{aligned}
\ddot{z}_{i j}+2 \zeta \dot{z}_{i j}+\alpha^{2} z_{i j}+\gamma \dot{\theta}_{i} \dot{\theta}_{j}-\frac{\theta_{i} \theta_{j}}{2}= & 0 \\
& (i, j=0,1, \ldots, N) .
\end{aligned}
$$

By using the useful relations (19), the necessary mean values can be calculated as

$$
\begin{aligned}
\left\langle\dot{z} \dot{\theta}^{2}\right\rangle & =\sum_{i=0}^{N} \sum_{j=0}^{N} \dot{z}_{i i} \dot{\theta}_{j}^{2}+2 \sum_{i=0}^{N} \sum_{j=0}^{N} \dot{z}_{i j} \dot{\theta}_{i} \dot{\theta}_{j} \\
\left\langle\dot{\theta}^{2}\right\rangle & =\sum_{j=0}^{N} \dot{\theta}_{j}^{2} .
\end{aligned}
$$

The iterative procedure of the implementation of $\mathrm{KL}$ expansion to partial linearization technique is shown in Figure 3.

\section{Numerical Demonstration}

This section will verify the accuracy of the proposed approach through an example of a ropeway gondola subjected to wind load. The values for parameters are selected to model a middle-size gondola as pendulum mass $m_{1}=790 \mathrm{~kg}$, pendulum length $l_{1}=3.8 \mathrm{~m}$, and structural damping $\zeta_{s}=1 \%$. 


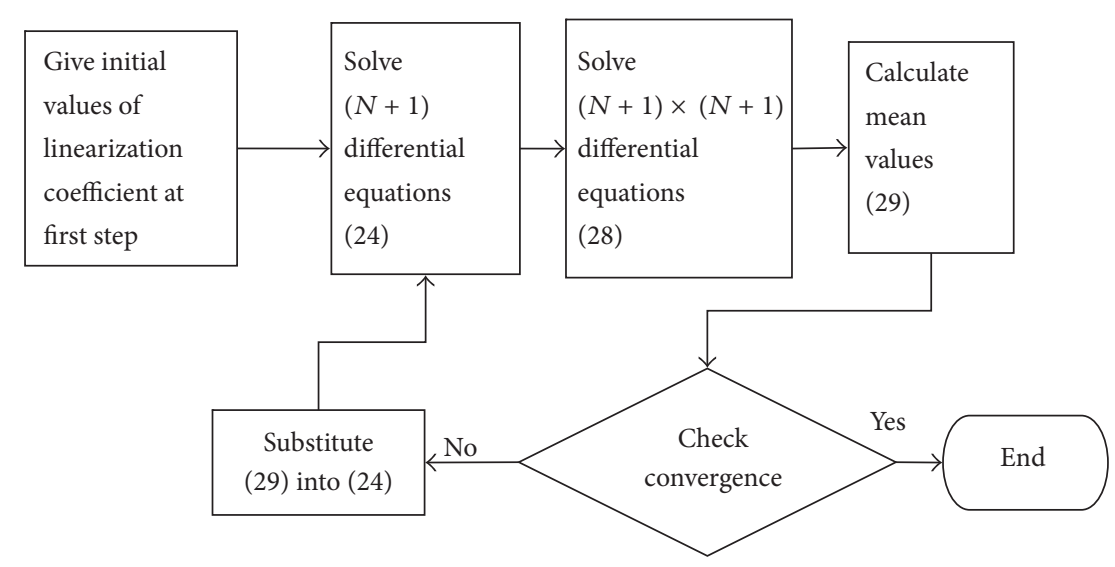

FIGURE 3: Flowchart of implementation of KL expansion to partial linearization technique.

5.1. Wind Force Model. The wind velocity contains two parts, the mean velocity and the fluctuating velocity. The fluctuating velocity $v(t)$ is taken from the Davenport spectrum [18]:

$$
S_{v}(\omega)=\frac{2 \kappa L^{2}}{\pi} \omega\left(1+\frac{L^{2} \omega^{2}}{4 \pi^{2} \bar{V}_{10}^{2}}\right)^{-4 / 3}
$$

in which $\omega$ is the frequency $(\mathrm{rad} / \mathrm{s}), \kappa$ is the surface drag coefficient (taken to be 0.005 ), $\bar{V}_{10}$ is the mean wind velocity at the standard height of 10 meters, and $L$ is the length scale (taken to be $1200 \mathrm{~m}$ ). The wind force $P(t)$ contains a static part and a dynamic part

$$
\begin{aligned}
P(t) & =\frac{1}{2} \rho C_{D} A\left(\bar{V}_{10}+v(t)\right)^{2} \\
& \approx \frac{1}{2} \rho C_{D} A \bar{V}_{10}^{2}+\rho C_{D} A \bar{V}_{10} v(t)
\end{aligned}
$$

in which $\rho$ is the air density (taken to be $1.3 \mathrm{~kg} / \mathrm{m}^{3}$ ), $C_{D}$ is the drag coefficient (taken to be 0.6 ), and $A$ is the structure's area exposed to wind (taken to be $3 \mathrm{~m}^{2}$ ). Because the DVA has only effect on the dynamic force, for demonstration purpose, let us consider only the dynamic term in (31). That means the external excitation $f(t)$ in (1) is taken by

$$
f(t)=\rho C_{D} A \bar{V}_{10} v(t) .
$$

The covariance function of $f(t)$ is calculated by

$$
\Gamma_{f f}(t, s)=\left(\rho C_{D} A \bar{V}_{10}\right)^{2} \int_{0}^{\infty} S_{v}(\omega) \cos (\omega(t-s)) d \omega
$$

The KL expansion (17) is obtained by discretizing the covariance function to make the covariance matrix $\Gamma_{f f}$. Then the discretized KL eigenvectors $\mathbf{f}_{j}$ and eigenvalue $\lambda_{j}$ can be obtained by solving the eigenvalue problem [14]:

$$
\Gamma_{f f} \mathbf{f}_{j}=\lambda_{j} \mathbf{f}_{j}
$$

In the numerical calculation, the time span $\left[0,30 T_{s}\right]$ is integrated with a time step $\Delta t=T_{s} / 10$, in which $T_{s}$ is the natural period of the undamped pendulum. This yields a size $301 \times 301$ for $\Gamma_{f f}$. The eigenvalues $\left\{\lambda_{j}\right\}_{j=1}^{100}$ in the case the mean velocity $\bar{V}_{10}=20 \mathrm{~m} / \mathrm{s}$ are shown in Figure 4 (a) while the eigenfunctions $\left\{f_{j}\right\}_{j=1}^{4}$ are plotted in Figure $4(\mathrm{~b})$.

5.2. Verification by Monte Carlo Simulations. Because there are no exact solutions of nonlinear systems (3) and (4), the Monte Carlo simulation is the only method that can be used to verify the accuracy of the proposed approach. The following DVA parameters are fixed: mass ratio $\mu=5 \%$ and location parameter $\gamma=1.5$. Two other parameters $\alpha$ and $\zeta$ and the wind velocity $\bar{V}_{10}$ have taken several values for comparison purpose. The number of samples of each Monte Carlo simulation is 5000. A major advantage of the KL expansion (17) is that only some large eigenvalues contribute significantly to the expression. In the numerical example in this paper, the calculated result presented in Figure 4(a) shows that the 100th eigenvalue only make a very small contribution $(=0.0245 \%)$ to the sum of all eigenvalues. To demonstrate the calculation procedure, we choose the number of KL eigenfunctions as 100 , which is very accurate to express the stochastic process.

Comparing with the Monte Carlo simulation, the partial linearization procedure presented in Figure 3 greatly decreases the computational time. This fact can be explained as follows. In each sample, the Monte Carlo method solves the nonlinear differential equations (3) and (4). Otherwise, for each eigenvector, the partial linearization solves the linear equations (24) and (28). For each time increment, the nonlinear differential equations (3) and (4) not only require much more numerical operation but also the calculations of trigonometric functions. On the Intel Atom CPU N450 of a cheap netbook, the partial linearization method (with 100 eigenvectors) takes 55.489 seconds to compute the time response (with 600 time steps) while the Monte Carlo method (with 5000 samples) requires 1485 seconds to complete. The computational time of the partial linearization method is only about $4 \%$ of that of the Monte Carlo method. 


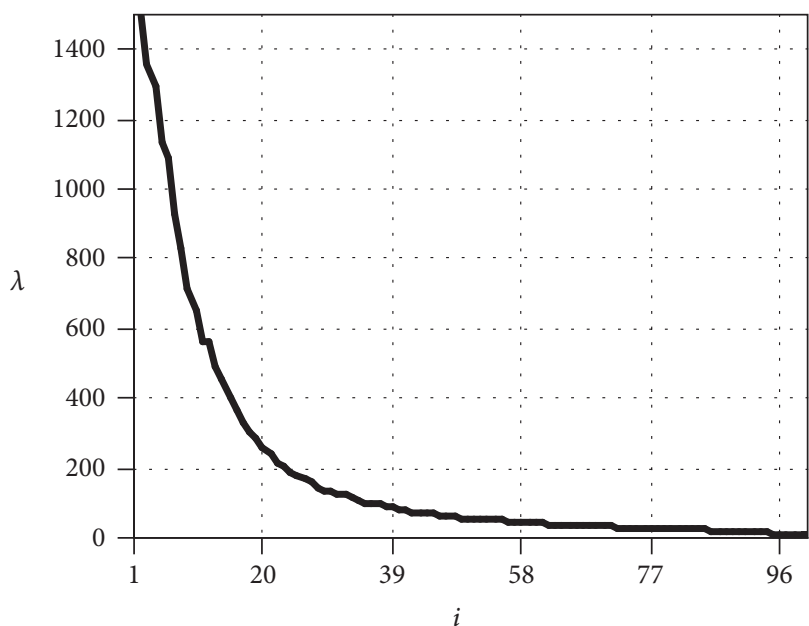

(a)

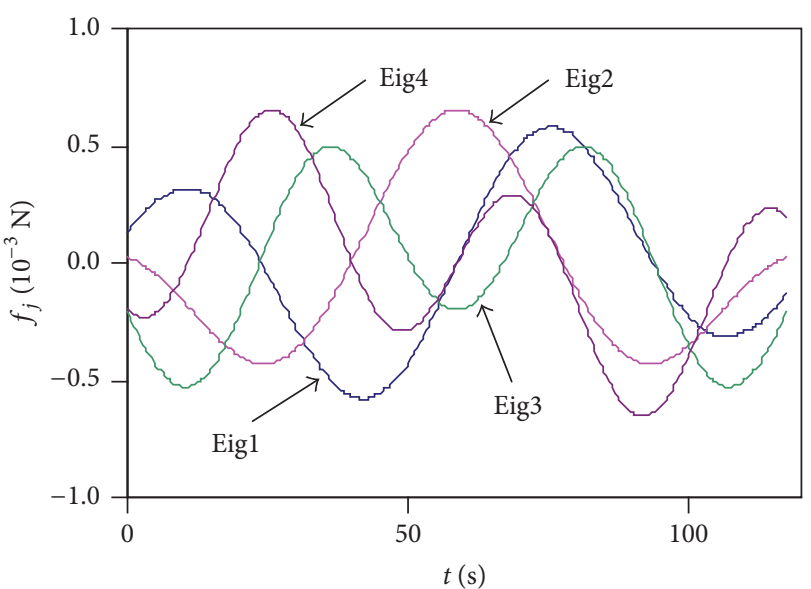

(b)

FIGURE 4: (a) 100 first eigenvalues and (b) 4 first eigenfunctions.
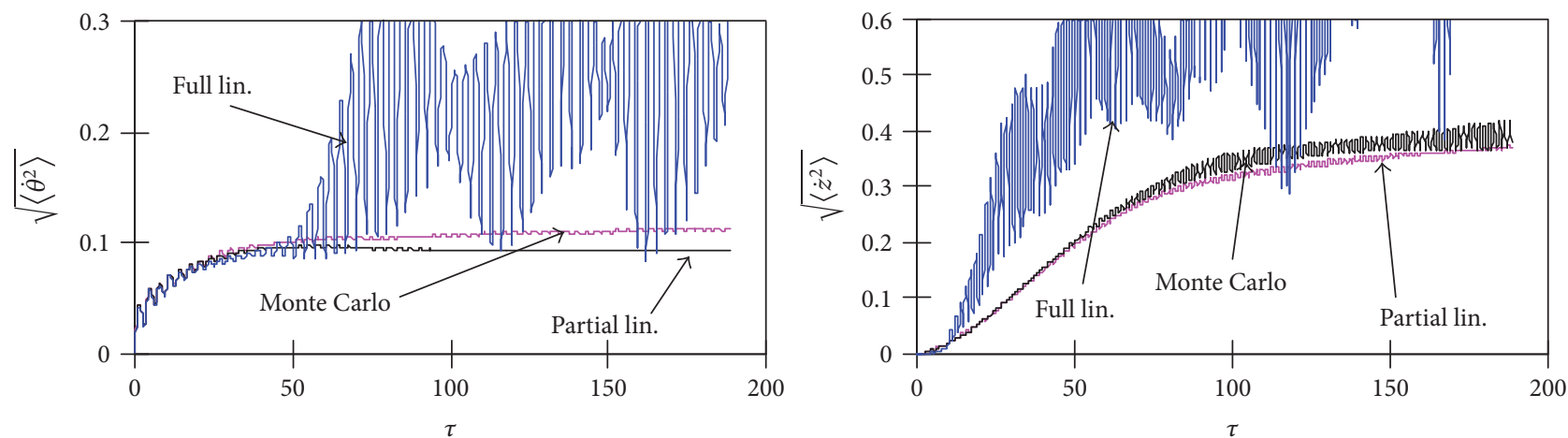

FIGURE 5: RMS values versus normalized time $\tau$ for the case $\bar{V}_{10}=20 \mathrm{~m} / \mathrm{s}, \alpha=2$, and $\zeta=0.01$.
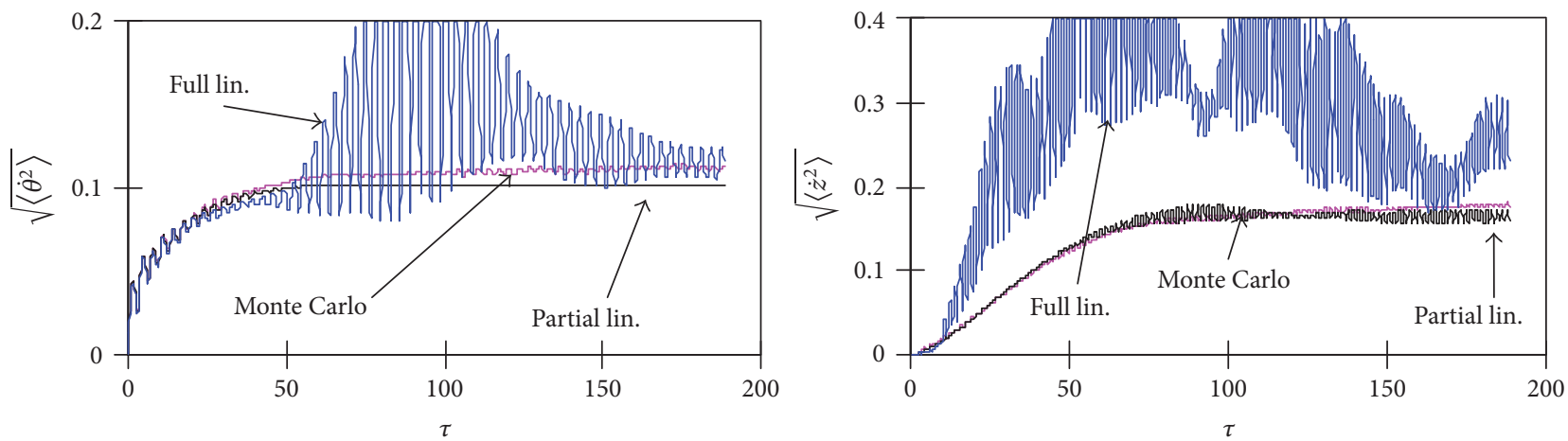

FIGURE 6: RMS values versus normalized time $\tau$ for the case $\bar{V}_{10}=20 \mathrm{~m} / \mathrm{s}, \alpha=2$, and $\zeta=0.05$.

The comparisons of the time histories are shown in Figures 5-10.

The results yield the following conclusions:

(i) In all cases, the partial linearization technique gives the solutions agreeing with the solutions of Monte Carlo simulation. In some cases (Figures 5 and 8), when the DVA velocities are large, the differences increase due to the effect of higher order terms in motion equations.

(ii) The DVA natural frequency is chosen near the resonant frequency $(\alpha=2)$. In this case, the DVA oscillates large and the full linearization technique totally fails to give the accuracy solutions in all cases. It simply cannot catch the stationary responses. 

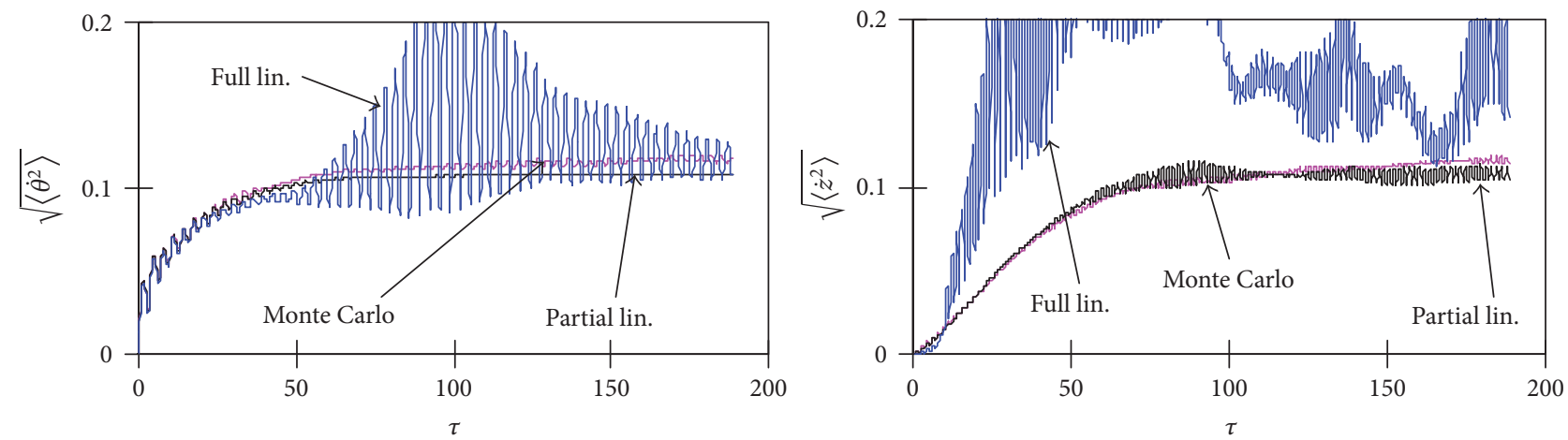

FIGURE 7: RMS values versus normalized time $\tau$ for the case $\bar{V}_{10}=20 \mathrm{~m} / \mathrm{s}, \alpha=2$, and $\zeta=0.1$.
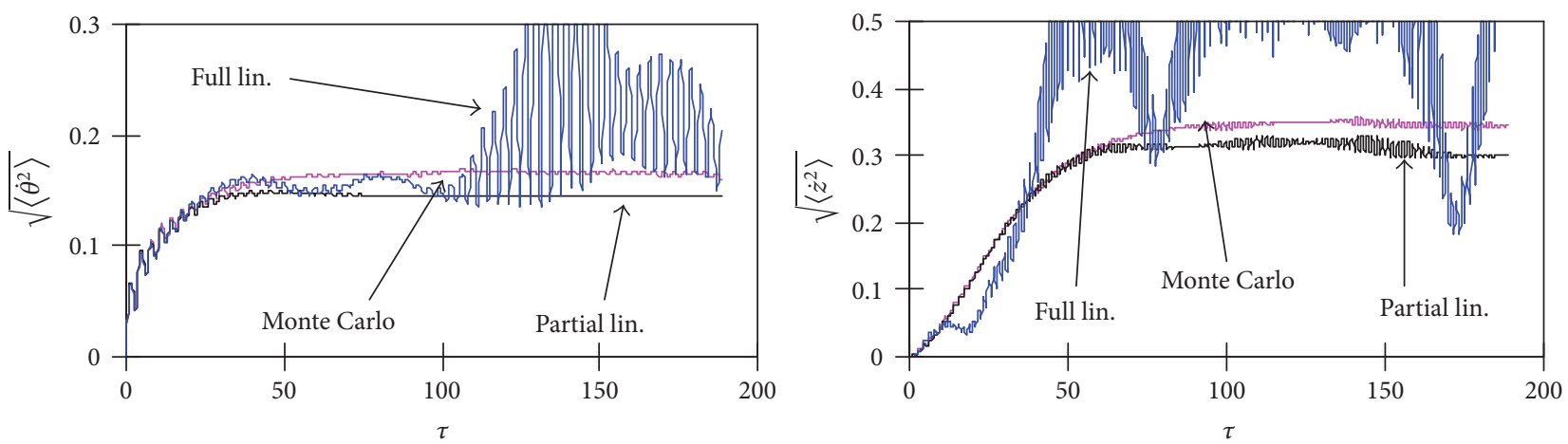

FIGURE 8: RMS values versus normalized time $\tau$ for the case $\bar{V}_{10}=25 \mathrm{~m} / \mathrm{s}, \alpha=2$, and $\zeta=0.05$.
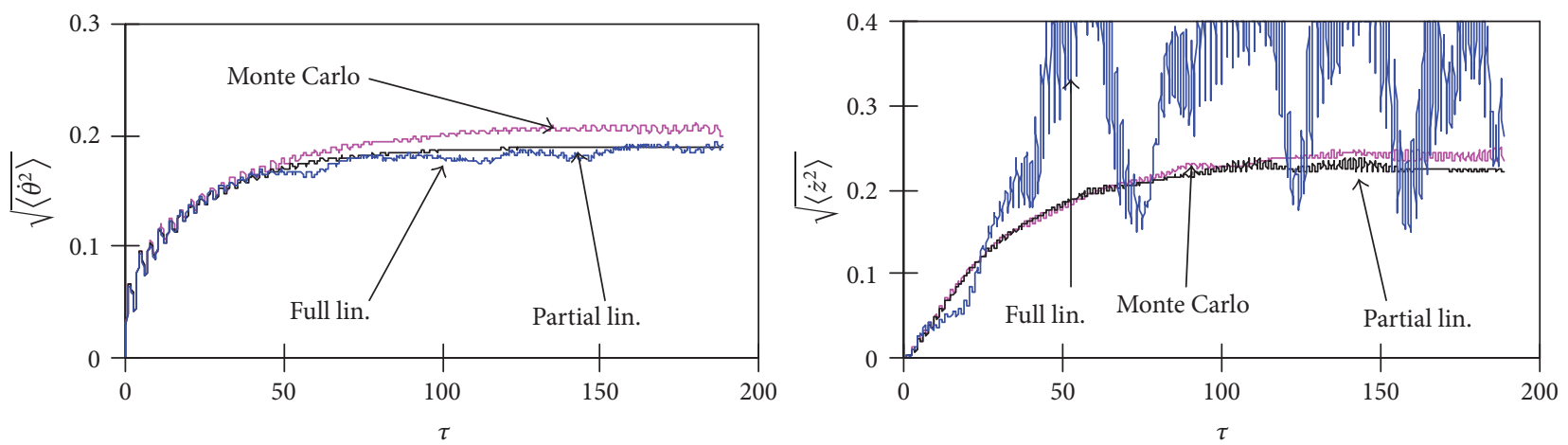

FIGURE 9: RMS values versus normalized time $\tau$ for the case $\bar{V}_{10}=25 \mathrm{~m} / \mathrm{s}, \alpha=1.8$, and $\zeta=0.05$.
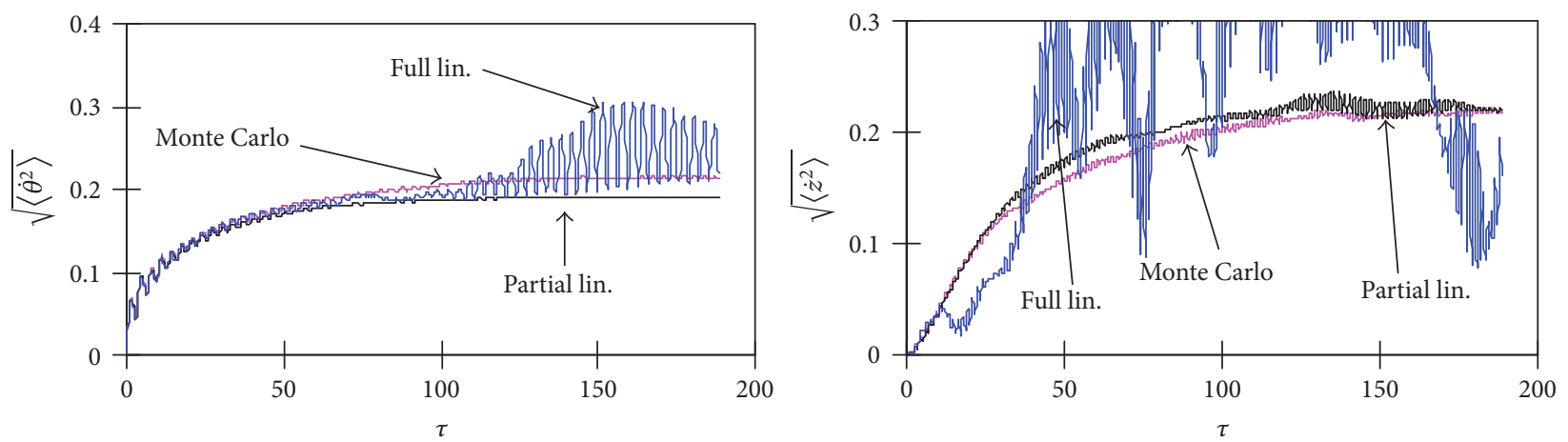

FIGURE 10: RMS values versus normalized time $\tau$ for the case $\bar{V}_{10}=25 \mathrm{~m} / \mathrm{s}, \alpha=2.2$, and $\zeta=0.05$. 
TABLE 1: Sweeping parameters.

\begin{tabular}{lccc}
\hline Parameter & $\begin{array}{c}\text { Interval } \\
\text { (from-to) }\end{array}$ & $\begin{array}{c}\text { Number of } \\
\text { points }\end{array}$ & Scale \\
\hline$\alpha$ & $1.8-2.2$ & 20 & $\begin{array}{c}\text { Linearly equally } \\
\text { spaced points } \\
\text { Logarithmically } \\
\text { equally spaced } \\
\text { points }\end{array}$ \\
\hline
\end{tabular}

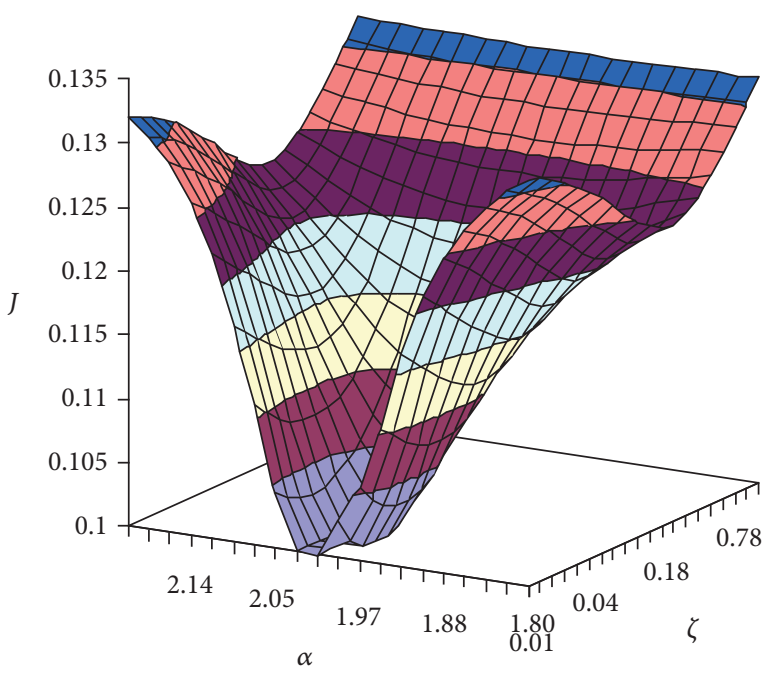

FIgURE 11: Performance index $J$ versus DVA parameters $\alpha$ and $\zeta$.

5.3. Stochastic Design. In comparison with the Monte Carlo simulation, the time required to process the procedure in Figure 3 is much shorter. Therefore, the proposed approach in this paper is quite suitable for the DVA design. For the demonstration purpose, let us consider the ropeway gondola model above. The design wind velocity is $\bar{V}_{10}=20 \mathrm{~m} / \mathrm{s}$. The performance index considered to be minimized has the form

$$
J=\sqrt{\left.\left\langle\dot{\theta}^{2}+\mu \dot{z}^{2}\right\rangle\right|_{\tau=\tau_{f}}}
$$

in which the normalized time $\tau_{f}$ is large enough to catch the stationary responses. The performance index $J$ is preferred to the pendulum's angle velocity itself because it can take into account the DVA responses. The larger value of $J$ means the poorer DVA performance. In design process, the data are collected by sweeping two parameters $\alpha$ and $\zeta$ through their intervals described in Table 1.

The total data size of $J$ is $20 \times 20=400$. The computation normalized time $\tau_{f}$ is chosen of 200 . The plots of $J$ versus two parameters $\alpha$ and $\zeta$ are shown in Figure 11.

It can be seen that the optimal value of the DVA frequency ratio $\alpha$ should be near to 2 . It is expected because the resonant frequency of the DVA is about twice that of the pendulum. This conclusion also agrees with the results in previous papers [4-6], which study the effects of harmonic excitation or initial conditions.

\section{Conclusions}

This paper considers the stochastic design of the pendulum structures attached with the dynamic vibration absorber using Coriolis force. The standard Gaussian equivalent linearization method applying to a simplified model fails to describe the system behaviors. Meanwhile, a partial linearization technique realized by the Karhunen-Loeve expansion works well in this simplified model. The proposed combination method is checked by the Monte Carlo simulations. The usefulness of the presented approach is demonstrated by a numerical example of a ropeway gondola induced by wind and by a design example of the dynamic vibration absorber.

\section{Appendix}

\section{Orthonormality Relation of Karhunen-Loeve Expansion}

Consider a continuous stochastic process $x(t)$ discretized at ordinarily equal intervals $\Delta t$, yielding a vector $\mathbf{x}$ defined as

$$
\mathbf{x}=\left[\begin{array}{lllll}
x(0) & x(\Delta t) & x(2 \Delta t) & \cdots & x(n \Delta t)
\end{array}\right]^{T} .
$$

The discrete KL expansion of $\mathbf{x}$ is defined as

$$
\mathbf{x}=\langle\mathbf{x}\rangle+\sum_{i=1}^{n+1} \xi_{i} \sqrt{\lambda_{i}} \psi_{i}
$$

in which $\psi_{i}$ and $\lambda_{i}$, respectively, are the eigenvectors and eigenvalues of the algebraic eigenvalue problem [14]:

$$
\begin{aligned}
& \Gamma_{x x} \psi_{i}=\lambda_{i} \psi_{i}, \\
& \psi_{i}^{T} \psi_{j}=\delta_{i j}
\end{aligned}
$$

in which $\Gamma_{x x}$ is the covariance matrix

$$
\boldsymbol{\Gamma}_{x x}=\left\langle(\mathbf{x}-\langle\mathbf{x}\rangle)(\mathbf{x}-\langle\mathbf{x}\rangle)^{T}\right\rangle
$$

and the Gaussian random variables $\xi_{i}$ are defined according to

$$
\xi_{i}=\frac{1}{\sqrt{\lambda_{i}}}(\mathbf{x}-\langle\mathbf{x}\rangle)^{T} \psi_{i}=\frac{1}{\sqrt{\lambda_{i}}} \psi_{i}^{T}(\mathbf{x}-\langle\mathbf{x}\rangle) .
$$

The following orthonormality relation can be obtained [14]:

$$
\begin{aligned}
\left\langle\xi_{j}\right\rangle & =0, \\
\left\langle\xi_{i} \xi_{j}\right\rangle & =\delta_{i j} \\
& (i, j=1, \ldots, N) .
\end{aligned}
$$

However, in this paper, we need to derive the orthonormality relation of higher orders of $\xi_{i}$, that is, the mean values $\left\langle\xi_{i} \xi_{j} \xi_{k}\right\rangle$ and $\left\langle\xi_{i} \xi_{j} \xi_{k} \xi_{l}\right\rangle$. We use the following expression of any Gaussian process [11, 12]:

$$
\langle(\mathbf{x}-\langle\mathbf{x}\rangle) g(\mathbf{x})\rangle=\Gamma_{x x}\left\langle\frac{\partial g(\mathbf{x})}{\partial \mathbf{x}}\right\rangle
$$


in which $g$ is any scalar function of vector $\mathbf{x}$. If we choose

$$
\begin{aligned}
g & =\xi_{j} \xi_{k}=(\mathbf{x}-\langle\mathbf{x}\rangle)^{T} \psi_{j}(\mathbf{x}-\langle\mathbf{x}\rangle)^{T} \psi_{k} \\
& =(\mathbf{x}-\langle\mathbf{x}\rangle)^{T} \boldsymbol{\psi}_{j} \psi_{k}^{T}(\mathbf{x}-\langle\mathbf{x}\rangle)
\end{aligned}
$$

then we have

$$
\frac{\partial g}{\partial \mathbf{x}}=\left(\psi_{j} \psi_{k}^{T}+\psi_{k} \psi_{j}^{T}\right)(\mathbf{x}-\langle\mathbf{x}\rangle)
$$

Using (A.5) gives

$$
\begin{aligned}
& \left\langle\xi_{i} \xi_{j} \xi_{k}\right\rangle=\frac{1}{\sqrt{\lambda_{i} \lambda_{j} \lambda_{k}}} \psi_{i}^{T}\langle(\mathbf{x}-\langle\mathbf{x}\rangle) g(\mathbf{x})\rangle \\
& =\frac{1}{\sqrt{\lambda_{i} \lambda_{j} \lambda_{k}}} \psi_{i}^{T} \Gamma_{x x}\left(\psi_{j} \psi_{k}^{T}+\psi_{k} \psi_{j}^{T}\right)\langle(\mathbf{x}-\langle\mathbf{x}\rangle)\rangle \\
& =0 .
\end{aligned}
$$

If we choose

$$
\begin{aligned}
g & =\xi_{j} \xi_{k} \xi_{l} \\
& =(\mathbf{x}-\langle\mathbf{x}\rangle)^{T} \psi_{j}(\mathbf{x}-\langle\mathbf{x}\rangle)^{T} \psi_{k}(\mathbf{x}-\langle\mathbf{x}\rangle)^{T} \psi_{l}
\end{aligned}
$$

then we have

$$
\begin{aligned}
\frac{\partial g}{\partial \mathbf{x}}= & \psi_{j}(\mathbf{x}-\langle\mathbf{x}\rangle)^{T} \psi_{k}(\mathbf{x}-\langle\mathbf{x}\rangle)^{T} \psi_{l} \\
& +\psi_{l}(\mathbf{x}-\langle\mathbf{x}\rangle)^{T} \psi_{j}(\mathbf{x}-\langle\mathbf{x}\rangle)^{T} \psi_{k} \\
& +\psi_{k}(\mathbf{x}-\langle\mathbf{x}\rangle)^{T} \psi_{l}(\mathbf{x}-\langle\mathbf{x}\rangle)^{T} \psi_{j}
\end{aligned}
$$

Because $\psi_{j}, \psi_{k}$, and $\psi_{l}$ are the eigenvectors of $\Gamma_{x x}$, from (A.3) we have

$$
\begin{aligned}
\left\langle\frac{\partial g}{\partial \mathbf{x}}\right\rangle & =\psi_{j} \psi_{k}^{T} \Gamma_{x x} \psi_{l}+\psi_{l} \psi_{j}^{T} \Gamma_{x x} \psi_{k}+\psi_{k} \psi_{l}^{T} \Gamma_{x x} \psi_{j} \\
& =\psi_{j} \lambda_{k} \delta_{k l}+\psi_{l} \lambda_{j} \delta_{j k}+\psi_{k} \lambda_{l} \delta_{l j} .
\end{aligned}
$$

Using (A.5) gives

$$
\begin{aligned}
& \left\langle\xi_{i} \xi_{j} \xi_{k} \xi_{l}\right\rangle=\frac{1}{\sqrt{\lambda_{i} \lambda_{j} \lambda_{k} \lambda_{l}}} \psi_{i}^{T}\left\langle(\mathbf{x}-\langle\mathbf{x}\rangle) \mathbf{g}^{T}(\mathbf{x})\right\rangle \\
& =\frac{1}{\sqrt{\lambda_{i} \lambda_{j} \lambda_{k} \lambda_{l}}} \\
& \cdot \psi_{i}^{T} \boldsymbol{\Gamma}_{x x}\left(\psi_{j} \lambda_{k} \delta_{k l}+\psi_{l} \lambda_{j} \delta_{j k}+\psi_{k} \lambda_{l} \delta_{l j}\right) .
\end{aligned}
$$

At last, the eigenvalue problem (A.3) is applied again to (A.14) to obtain

$$
\left\langle\xi_{i} \xi_{j} \xi_{k} \xi_{l}\right\rangle=\delta_{i j} \delta_{k l}+\delta_{i k} \delta_{j l}+\delta_{i l} \delta_{j k}
$$

The orthonormality relations (A.10) and (A.15) are rewritten in (19) to realize the partial linearization technique.

\section{Conflicts of Interest}

The author declares that there are no conflicts of interest regarding the publication of this paper.

\section{Acknowledgments}

This research is funded by Vietnam National Foundation for Science and Technology Development (NAFOSTED) under Grant no. "107.01-2015.35."

\section{References}

[1] G. B. Warburton, "Optimum absorber parameters for various combinations of response and excitation parameters," Earthquake Engineering \& Structural Dynamics, vol. 10, no. 3, pp. 381401, 1982.

[2] H. Matsuhisa, R. Gu, Y. Wang, O. Nishihara, and S. Sato, "Vibration control of a ropeway carrier by passive dynamic vibration absorbers," JSME International Journal, Series C: Dynamics, Control, Robotics, Design and Manufacturing, vol. 38, no. 4, pp. 657-662, 1995.

[3] H. Matsuhisa and M. Yasuda, "Location effect of dynamic absorbers on rolling structures," in Proceedings of the AsiaPacific Vibration Conference, pp. 439-444, Gold Coast, Australia, 2003.

[4] H. Matsuhisa, H. Kitaura, M. Isono, H. Utsuno, J. G. Park, and M. Yasuda, "A new Coriolis dynamic absorber for reducing the swing of gondola," in Proceedings of the Asia-Pacific Vibration Conference, pp. 211-215, Langkawi, Malaysia, 2005.

[5] L. D. Viet, N. D. Anh, and H. Matsuhisa, “The effective damping approach to design a dynamic vibration absorber using Coriolis force," Journal of Sound and Vibration, vol. 330, no. 9, pp. 19041916, 2011.

[6] L. D. Viet, N. D. Anh, and H. Matsuhisa, "Vibration control of a pendulum structure by a dynamic vibration absorber moving in both normal and tangential directions," Proceedings of the Institution of Mechanical Engineers, Part C: Journal of Mechanical Engineering Science, vol. 225, no. 5, pp. 1087-1095, 2011.

[7] H. Janocha, Ed., Adaptronics and Smart Structures: Basics Materials Design and Applications, Springer, 2007.

[8] O. Nishihara, H. Matsuhisa, and S. Sato, "Vibration damping mechanisms with gyroscopic moments," JSME International Journal, Series III, vol. 35, no. 1, pp. 50-55, 1992.

[9] C. Proppe, H. J. Pradlwarter, and G. I. Schuëller, "Equivalent linearization and Monte Carlo simulation in stochastic dynamics," Probabilistic Engineering Mechanics, vol. 18, no. 1, pp. 1-15, 2003.

[10] L. Socha, Linearization Methods for Stochastic Dynamic Systems, vol. 730 of Lecture Notes in Physics, Springer, Berlin, Germany, 2008.

[11] L. D. Lutes and S. Sarkani, Random Vibration: Analysis of Structural and Mechanical Systems, Elsevier, Amsterdam, The Netherlands, 2004.

[12] T. S. Atalik and S. Utku, "Stochastic linearization of multidegree-of-freedom non-linear systems," Earthquake Engineering \& Structural Dynamics, vol. 4, no. 4, pp. 411-420, 1976.

[13] L. D. Viet, "Partial stochastic linearization of a spherical pendulum with coriolis damping produced by radial spring and damper," Journal of Vibration and Acoustics, Transactions of the ASME, vol. 137, no. 5, Article ID 054504, 2015. 
[14] C. A. Schenk and G. I. Schueller, Uncertainty Assessment of Large Finite Element Systems, vol. 24, Springer, Berlin, Germany, 2005.

[15] P. B. Gonçalves, F. M. A. Silva, and Z. J. G. N. Del Prado, "Lowdimensional models for the nonlinear vibration analysis of cylindrical shells based on a perturbation procedure and proper orthogonal decomposition," Journal of Sound and Vibration, vol. 315, no. 3, pp. 641-663, 2008.

[16] K. J. Craig and W. J. Roux, "On the investigation of shell buckling due to random geometrical imperfections implemented using Karhunen-Loève expansions," International Journal for Numerical Methods in Engineering, vol. 73, no. 12, pp. 1715-1726, 2008.

[17] H. J. Pradlwarter and G. I. Schuëller, "Uncertain linear structural systems in dynamics: efficient stochastic reliability assessment," Computers and Structures, vol. 88, no. 1-2, pp. 74-86, 2010.

[18] A. G. Davenport, "The spectrum of horizontal gustiness near the ground in high winds," Quarterly Journal of the Royal Meteorological Society, vol. 87, no. 372, pp. 194-211, 1961. 


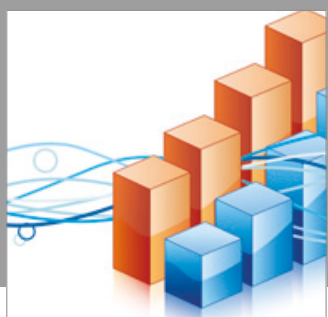

Advances in

Operations Research

vatersals

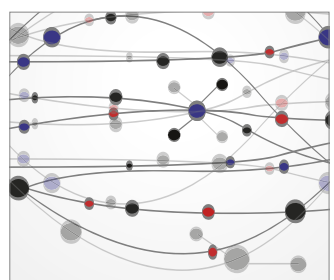

\section{The Scientific} World Journal
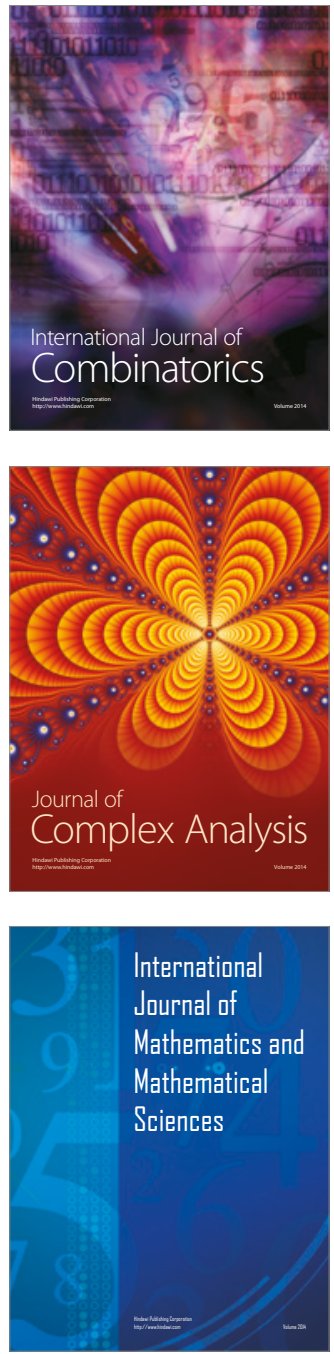
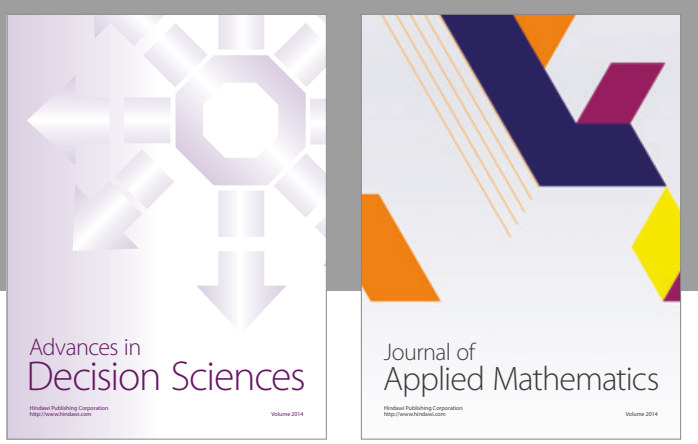

Algebra

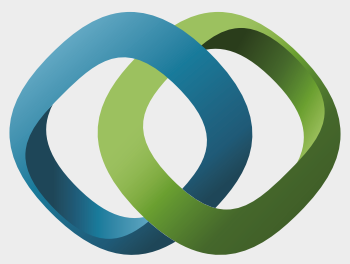

\section{Hindawi}

Submit your manuscripts at

https://www.hindawi.com
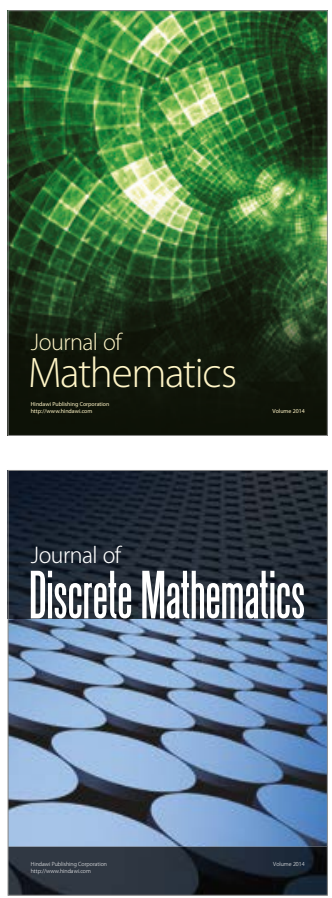

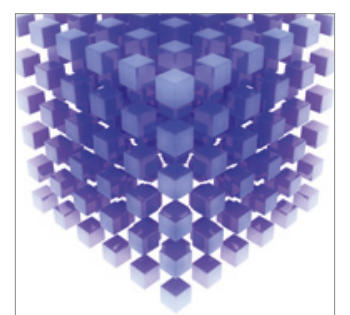

Mathematical Problems in Engineering
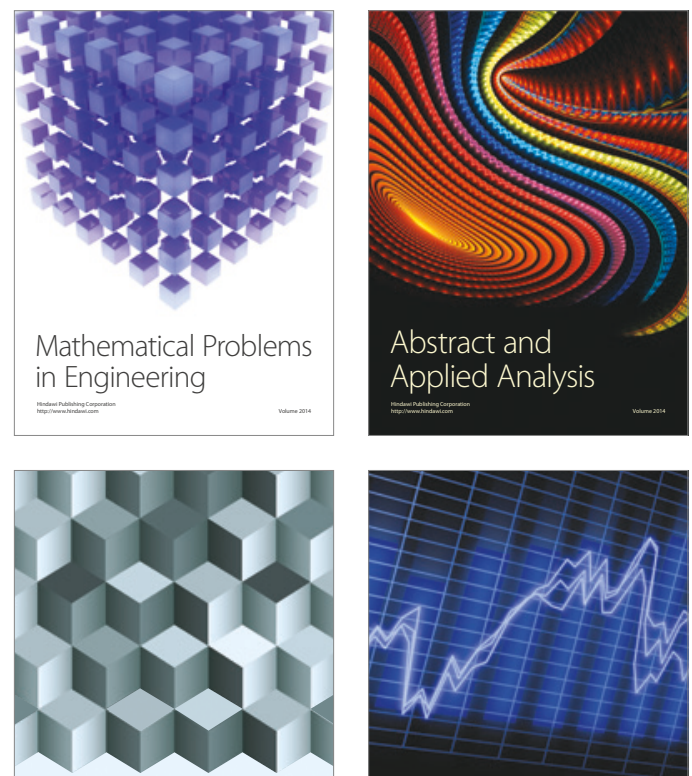

Journal of

Function Spaces

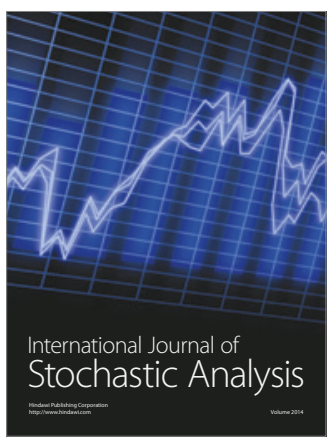

Probability and Statistics
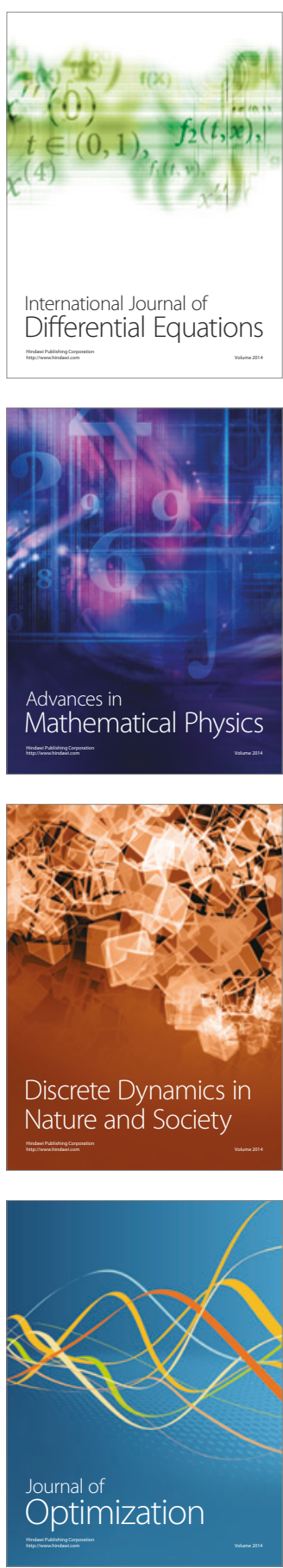